\title{
Association between Cardiovascular Burden and Requirement of Intensive Care among Patients with Mild COVID-19
}

\author{
Shi Tai $₫$, 1,2 Jianjun Tang, ${ }^{1,2}$ Bilian Yu, ${ }^{1,2}$ Liang Tang, ${ }^{1,2}$ Yang Wang, ${ }^{2}$ Huilin Zhang, \\ Weihong Zhu, ${ }^{2}$ Kui Xiao, ${ }^{2}$ Chuan Wen, ${ }^{2}$ Chongqin Tan, ${ }^{2}$ Zhongbiao Jiang, ${ }^{2}$ \\ Chuanhao Jiang, ${ }^{2}$ Li Zhu, ${ }^{2}$ Li Jiang, ${ }^{2}$ Qiming Liu, ${ }^{1}$ Xinqun Hu $\mathbb{D}^{1},{ }^{1}$ Zhenfei Fang $\mathbb{D}^{1}{ }^{1}$ \\ Xuping Li, ${ }_{1}^{1}$ Jiaxing Sun, ${ }^{1}$ Zhaowei Zhu, ${ }^{1}$ Hui Yang, ${ }^{1}$ Tao Tu, ${ }^{1}$ Yichao Xiao, ${ }^{1}$ Mingxian Chen, ${ }^{1}$ \\ Yuhu He, ${ }^{1}$ Xiangping Chai $\mathbb{D}^{3,2}$ Junmei Xu $\mathbb{D}^{4},{ }^{4}$ and Shenghua Zhou $\mathbb{D}^{1}$ \\ ${ }^{1}$ Department of Cardiovascular Medicine, The Second Xiangya Hospital of Central South University, Changsha, China \\ ${ }^{2}$ National Emergency Medical Team, China \\ ${ }^{3}$ Department of Emergency Medicine, The Second Xiangya Hospital of Central South University, Changsha, China \\ ${ }^{4}$ Department of Anesthesiology, The Second Xiangya Hospital of Central South University, Changsha, China
}

Correspondence should be addressed to Junmei Xu; 13975148864@139.com and Shenghua Zhou; zhoushenghua@csu.edu.cn

Received 29 April 2020; Revised 10 June 2020; Accepted 26 June 2020; Published 16 August 2020

Academic Editor: Leonardo De Luca

Copyright (C) 2020 Shi Tai et al. This is an open access article distributed under the Creative Commons Attribution License, which permits unrestricted use, distribution, and reproduction in any medium, provided the original work is properly cited.

\begin{abstract}
Background. Information regarding the impact of cardiovascular (CV) conditions on disease progression among patients with mild coronavirus disease 2019 (COVID-19) is limited. Methods. This study evaluated the association of underlying CV conditions with disease progression in patients with mild COVID-19. The primary outcome was the need to be transferred to the designated hospital for intensive care due to COVID-19 disease progression. The patients were divided into with and without CV conditions as well as stable and intensive care groups. Results. Of the 332 patients with mild COVID-19, the median age was 51 years (IQR, 40-59 years), and 200 (61.2\%) were female. Of the 48 (14.5\%) patients with CV conditions, 23 (47.9\%) progressed to severe disease status and required intensive care. Compared with patients without CV conditions, patients with CV conditions were older and more likely to have fatigue, chest tightness, and myalgia. The rate of requiring intensive care was significantly higher among patients with CV conditions than in patients without CV conditions $(47.92 \%$ vs. $12.4 \%$; $P<0.001)$. In subgroup analysis, the rate of requiring intensive care was also higher among patients with either hypertension or coronary heart disease (CHD) than in patients without hypertension or CHD. The multivariable regression model showed that CV condition served as an independent risk factor for intensive care (odds ratio (OR), 2.652 (95\% CI, 1.019-6.899)) after adjustment for various cofounders. Conclusions. Patients with mild COVID-19 complicating CV conditions are susceptible to develop severe disease status and requirement for intensive care.
\end{abstract}

\section{Introduction}

The outbreak of coronavirus disease 2019 (COVID-19), which is caused by severe acute respiratory syndrome coronavirus 2 (SARS-CoV-2) virus [1], rapidly progressed to a pandemic and was declared a public health concern [2]. Genetic sequencing of the virus suggests that SARS-CoV-2 is a betacoronavirus closely linked to the SARS virus. While most people with COVID-19 develop mild or uncomplicated illness, approximately $14 \%$ of the people develop severe disease and finally require oxygen support; unfortunately, $5 \%$ of the patients even need to be treated in the intensive care unit $[3,4]$. Importantly, a large proportion of affected patients have been reported to have underlying cardiovascular diseases (CVD) [5-7] and can present additional challenge in the battle against outbreaks of novel virus infections. However, information on factors affecting disease progression among patients with mild COVID-19 is limited.

Coronaviruses are known to affect the cardiovascular system $[8,9]$, and the prevalence of underlying CVD is common 
and steadily increasing in China during recent decades, which may serve as a risk factor to the susceptibility and severity of infectious diseases [10]. Although most mild patients with COVID-19 are thought to have a favorable prognosis, those with chronic underlying conditions may suffer from worse outcomes [11]. Nevertheless, there is sparse data on clinical presentation of COVID-19 in specific populations, such as mild illness and coexisting CVD. In the present study, we report the clinical characteristics and factors associated with developing severe disease after admission in patients hospitalized into temporary hospitals [12] with mild COVID-19.

\section{Methods}

2.1. Study Participants and Criteria for Patient Admission, Discharge, and Referral. Consecutive patients admitted to Wuchang temporary hospital with laboratory-confirmed COVID-19 from February 5, 2020, to March 10, 2020, were included in this retrospective cohort study. The criteria for patient admission, discharge, and referral were established according to the World Health Organization interim guidance [4] and Guidance for Corona Virus Disease 2019: Prevention, Control, Diagnosis and Management (Tentative Sixth Edition) [13].

Patients who met all the following conditions were discharged alive from the hospital: (1) normal temperature for more than 3 consecutive days, (2) significantly improved respiratory symptoms, (3) resting $\mathrm{SpO}_{2}>93 \%$, (4) radiology examination suggesting a significantly reduced pulmonary inflammation, and (5) negative results of two consecutive SARS-CoV-2 testing by real-time reverse transcriptionpolymerase chain (RT-PCR) within a 24-hour interval.

Patients with one of the following conditions were referred to the designated hospital for intensive care: (1) respiratory rate $\geq 30$ breaths per minute; (2) resting $\mathrm{SpO}_{2}$ $\leq 93 \%$; (3) temperature $\geq 38.5^{\circ} \mathrm{C}$ for more than 2 consecutive days even with proper treatment; (4) severe dysfunction of the heart, liver, lung, kidney, or brain; and (5) other emergent conditions.

This study was approved by the Institutional Review Board at the Second Xiangya Hospital of Central South University and Renmin Hospital of Wuhan University. The need for written informed consent was waived by the committee.

2.2. Data Collection. The demographic characteristics (age and sex) and clinical data (symptoms, comorbidities, laboratory findings, and outcomes) for participants during hospitalization were collected from electronic medical records by 2 investigators. The radiologic assessments included chest radiography or computed tomography. All data were independently reviewed and entered into the computer database by 2 analysts. Cardiovascular disease (CVD) is a group of disorders of the heart and blood vessels and includes coronary heart disease (CHD), cerebrovascular disease, peripheral arterial disease, rheumatic and congenital heart diseases, and venous thromboembolism $[14,15]$. According to the previous studies $[16,17]$, the present study includes hypertension and
CVD. The patients were divided into with and without $\mathrm{CV}$ conditions as well as stable and intensive care groups.

2.3. Treatment. All patients with mild COVID-19 were treated according to the Guidance for Corona Virus Disease 2019: Prevention, Control, Diagnosis and Management (Tentative Sixth Edition). Antiviral therapy with umifenovir (0.2 g, three times daily for 5 days) or oseltamivir ( $75 \mathrm{mg}$, twice daily for 5 days) was administered. Traditional Chinese medicine with the Pneumonia Number 1 Decoction or Pneumonia Number 2 Decoction was also used for 3 days as a cycle until the symptoms were relieved. Statins, beta-blockers, and aspirin were administered for patients with CHD; nifedipine GITS was administered for patients with hypertension.

2.4. Outcome. The clinical outcomes (i.e., discharge alive and referral to the designated hospital for intensive care) were monitored up to March 25, 2020, the final date of follow-up. The primary end point was the need to be transferred to the designated hospital due to COVID-19 developing severe disease status and requirement for intensive care. Successful treatment toward hospital discharge comprised relieved clinical symptoms, normal body temperature, significant resolution of inflammation as shown by chest radiography, and at least 2 consecutive negative results shown by RT-PCR assay for SARS-CoV-2.

2.5. Statistical Analysis. Descriptive statistics were obtained for all study variables. All categorical variables were compared for the study outcome by using Fisher's exact test or $\chi^{2}$ test, and continuous variables were compared using the $t$-test or the Mann-Whitney $U$ test, as appropriate. Continuous data are expressed as mean (SD) or median (interquartile range (IQR)) values. Categorical data are expressed as proportions. Survival curves were plotted using the Kaplan-Meier method and compared between patients with and without CV conditions using the log-rank test. Multivariate logistic regression models were used to determine the independent risk factors for referral during hospitalization. Data were analyzed using Stata15. Statistical charts were generated using Excel 2016 (Microsoft) or Stata15. For all the statistical analyses, $P<0.05$ was considered significant.

\section{Results}

3.1. Demographics and Characteristics. Figure 1 shows the flowchart for patient recruitment. Briefly, of all 394 patients in the medical record system, who were screened initially from February 5, 2020, to March 10, 2020, 43 patients without available medical information and duplicated records and 19 patients who transferred to the designated hospital because of non-COVID-19 clinical factors were excluded (8 patients requested for referral by themselves, 6 patients with anxiety and insomnia, 1 patient with manic depression, 1 patient with dysgnosia, 1 patient with chickenpox, 1 patient with acute renal colic, and 1 patient with lumbar disc herniation). Finally, the study population consisted of 332 patients hospitalized with confirmed COVID-19: 58 patients (17.5\%) required referral because of requirement for intensive care (intensive group) and 274 patients (82.5\%) were discharged 


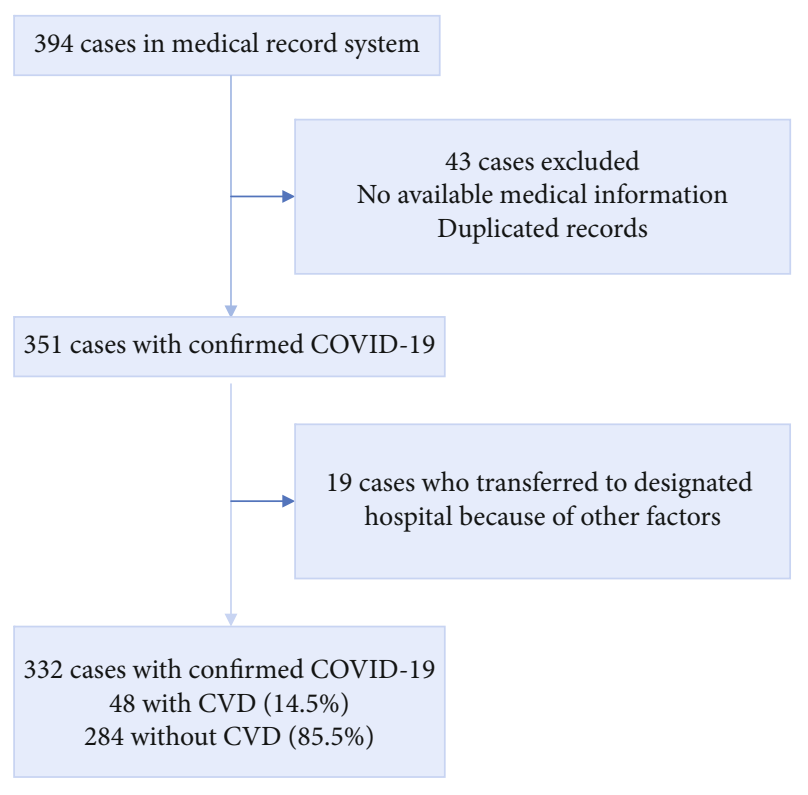

Figure 1: Flowchart of patient recruitment.

(stable group). The median age was 51 years (median (IQR) age, IQR, 40-59 years), and 200 (61.2\%) were female. Among these patients, fever (177 patients (63.2\%)) was the most common symptom. Cough, fatigue, chest tightness, and myalgia were present in 108 patients (38.4\%), 32 patients (11.5\%), 39 patients (13.9\%), and 12 patients (4.3\%), respectively. Diarrhea (11 patients (3.9\%)), sore throat (4 patients $(1.4 \%)$ ), rhinorrhea (4 patients $(1.4 \%)$ ), headache (7 patients $(2.5 \%)$ ), and toothache (2 patients $(0.6 \%))$ were rare. Hypertension (37 patients (11.1\%)) and diabetes (11 patients (3.3\%)) were the most common coexisting conditions. Table 1 presents the clinical profile of stable patients and intensive care patients. Results showed that prevalence of chest tightness, toothache, CHD, hypertension, and twice or more positive SARS-CoV-2 testing was significantly higher, while positive radiology findings was significantly lower in the intensive care group than in the stable group.

Compared with patients without $\mathrm{CV}$ conditions, patients with CV conditions were older (56 (45-62) years vs. 50 (3959) years; $P=0.007$ ) and more likely to have fatigue (13 of 48 patients $(28.3 \%)$ vs. 26 of 284 patients $(11.1 \%) ; P=0.002)$, chest tightness (18 of 48 patients $(40.0 \%)$ vs. 14 of 284 patients $(6.0 \%) ; P<0.001$ ), and myalgia (6 of 48 patients $(13.0 \%)$ vs. 6 of 284 patients $(2.6 \%) ; P=0.001)$. Moreover, comorbidities including diabetes $(4(8.3 \%)$ vs. $7(2.5 \%))$ and lung disease $(4(8.3 \%)$ vs. $3(1.1 \%))$ were present more often among patients with CV conditions (all $P<0.05)$ (Table 2). In subgroup analysis, compared with patients without hypertension, patients with hypertension were older (56 (49-60) years vs. 50 (39-59) years; $P=0.009)$ and more likely to have fatigue (10 of 38 patients $(27.0 \%)$ vs. 29 of 294 patients (11.9\%); $P=0.014$ ) and chest tightness (14 of 38 patients $(38.9 \%)$ vs. 18 of 294 patients $(7.4 \%) ; P<0.001)$. Moreover, comorbidities including diabetes (4 (10.5\%) vs. $7(2.4 \%) ; P=0.027)$ were present more often among patients with hypertension. Compared with patients without CHD, patients with CHD were more likely to have fatigue ( 5 of 11 patients (45.5\%) vs. 34 of 321 patients (12.6\%); $P=0.002)$ and chest tightness (6 of 11 patients (54.5\%) vs. 26 of 321 patients $(9.7 \%) ; P<0.001)$. Lung disease $(2(18.2 \%)$ vs. 5 $(1.6 \%) ; P=0.019)$ was present more often among patients with CHD (Supplemental Table 1).

3.2. Laboratory and Radiographic Findings. In terms of laboratory findings, there were no significantly statistic differences between patients with and without CVD, including white cell count, neutrophil count, neutrophil to lymphocyte ratio (NLR), lymphocyte count, platelet count, and C-reactive protein. Results of SARS-CoV-2 testing and radiologic findings were similar between the 2 groups. There seemed to be lower radiologic abnormality observed in patients with $\mathrm{CV}$ conditions than those without CV conditions (52.8\% vs. $67.2 \%)$ on admission. Importantly, there were more patients with progression of radiologic findings in the $\mathrm{CV}$ condition group than in the without CV condition group (7.1\% vs. $2.6 \%)$, suggesting that the need for referral to the designated hospital for intensive care is linked to the progression of COVID-19.

3.3. Clinical Outcome. Of the 332 patients, 48 (14.5\%) had underlying CV conditions including hypertension and CHD, and 23 (47.9\%) developed severe diseases who required intensive care. The median time from symptom onset to admission in the temporary hospital was 12 days (IQR, 8-16 days), and the median time from admission in the temporary hospital to referral to the designated hospital was 14 days (IQR, 10-19 days). Patients with CV conditions vs. those without $\mathrm{CV}$ conditions had longer durations from symptom onset to admission (mean, 14 (range, 8-18) days vs. 12 (range, 8-15) days) and duration from admission to referral (15 (IQR, 8-20) days vs. 14 (IQR, 10-19) days). As shown in Table 2 and the Kaplan-Meier curves in Figure 2, the requirement for intensive care was higher among patients with vs. without $\mathrm{CV}$ conditions (23 of $48(47.9 \%)$ vs. 35 of $284(12.4 \%) ; P<0.001)$ and discharge rate was lower in patients with $\mathrm{CV}$ conditions vs. without CV conditions (25 of $48(52.1 \%)$ vs. 249 of 284 (87.6\%); $P<0.001)$. In subgroup analysis, the requirement for intensive care rate was higher among patients with vs. without either hypertension or $\mathrm{CHD}$ and discharge rate was lower in patients with vs. without either hypertension or CHD as shown in Supplemental Table 1 and Kaplan-Meier curves in Supplemental Figures 1 and 2. As shown in Supplemental Table 2, chest tightness, platelet count, radiologic abnormality, and more than once positive SARSCoV-2 testing by RT-PCR were risk factors of intensive care in this patient cohort. To evaluate whether $\mathrm{CV}$ conditions are associated with requirement for intensive care in patients with mild COVID-19, we adjusted for demographic characteristics, comorbidities, and clinical profiles: model 1 adjusted for age and sex; model 2 adjusted for age, sex, chest tightness, diabetes mellitus, and lung diseases; and model 3 adjusted for age, sex, chest tightness, diabetes mellitus, lung diseases, platelet count, radiologic abnormality, and more than once positive SARS-CoV-2 testing by RT-PCR. As shown in Table 3, CV condition remains as an independent risk factor for requirement for intensive care in patients with 
TABLE 1: Clinical features of patients with mild COVID-19.

\begin{tabular}{|c|c|c|c|c|}
\hline Characteristic & $\begin{array}{l}\text { All patients } \\
(n=332)\end{array}$ & $\begin{array}{c}\text { Stable group } \\
(n=274,82.5 \%)\end{array}$ & $\begin{array}{l}\text { Intensive care group } \\
\quad(n=58,17.5 \%)\end{array}$ & $P$ value \\
\hline \multicolumn{5}{|l|}{$\operatorname{Sex}$} \\
\hline Male, $n(\%)$ & $132(39.8)$ & $105(38.3)$ & $27(46.6)$ & 0.245 \\
\hline Female, $n(\%)$ & $200(61.2)$ & $169(61.7)$ & $31(53.4)$ & 0.245 \\
\hline Age, median (IQR) & $51(40-59)$ & $50.5(40-59)$ & $52(42-60)$ & 0.478 \\
\hline \multicolumn{5}{|l|}{ Symptoms } \\
\hline Fever, $n(\%)$ & $177(63.2)$ & $142(63.4)$ & $35(62.5)$ & 0.901 \\
\hline Cough, $n(\%)$ & $108(38.4)$ & $88(39.1)$ & $20(35.7)$ & 0.640 \\
\hline Fatigue, $n(\%)$ & $39(13.9)$ & $28(12.5)$ & $11(19.6)$ & 0.167 \\
\hline Chest tightness, $n(\%)$ & $32(11.5)$ & $15(6.7)$ & $17(29.3)$ & $<0.001$ \\
\hline Diarrhea, $n(\%)$ & $11(3.9)$ & $8(3.6)$ & $3(5.4)$ & 0.538 \\
\hline Headache, $n(\%)$ & $7(2.5)$ & $6(2.7)$ & $1(1.8)$ & 0.702 \\
\hline Sore throat, $n(\%)$ & $4(1.4)$ & $2(0.9)$ & $2(3.4)$ & 0.181 \\
\hline Rhinorrhea, $n$ (\%) & $4(1.4)$ & $4(1.8)$ & $0(0.0)$ & 0.587 \\
\hline Myalgia, $n(\%)$ & $12(4.3)$ & $9(4.0)$ & $3(5.4)$ & 0.658 \\
\hline Toothache, $n(\%)$ & $2(0.6)$ & $0(0.0)$ & $2(3.4)$ & 0.030 \\
\hline \multicolumn{5}{|l|}{ Comorbidities } \\
\hline CV conditions, $n(\%)$ & $48(14.5)$ & $25(7.5)$ & $23(39.7)$ & $<0.001$ \\
\hline $\mathrm{CHD}, n(\%)$ & $11(3.3)$ & $1(0.4)$ & $10(17.2)$ & $<0.001$ \\
\hline Hypertension, $n(\%)$ & $38(11.4)$ & $21(7.7)$ & $17(27.9)$ & $<0.001$ \\
\hline Diabetes, $n(\%)$ & $11(3.3)$ & $7(2.6)$ & $4(6.9)$ & 0.106 \\
\hline Lung disease, $n(\%)$ & $7(2.1)$ & $4(1.5)$ & $3(5.2)$ & 0.105 \\
\hline Radiologic abnormality, $n$ (\%) & $201(65.5)$ & $183(70.1)$ & $18(36.7)$ & $<0.001$ \\
\hline \multicolumn{5}{|l|}{ Positive SARS-CoV-2 testing by RT-PCR } \\
\hline Twice or more, $n(\%)$ & $72(23.0)$ & $48(18.3)$ & $24(48.0)$ & $<0.001$ \\
\hline Once, $n(\%)$ & $241(77.0)$ & $215(81.7)$ & $26(74)$ & $<0.001$ \\
\hline \multicolumn{5}{|l|}{ Laboratory findings } \\
\hline Red blood cell count $\left(* 10^{12} / \mathrm{L}\right)$, median (IQR) & $4.39(4.05-4.73)$ & $4.39(4.05-4.73)$ & $4.42(4.02-4.76)$ & 0.863 \\
\hline White cell count $\left(* 10^{9} / \mathrm{L}\right)$, median (IQR) & $5.25(4.49-6.37)$ & $5.19(4.47-6.33)$ & $5.62(4.88-6.58)$ & 0.058 \\
\hline Neutrophil count $\left(* 10^{9} / \mathrm{L}\right)$, median (IQR) & $3.21(2.57-4.08)$ & $3.18(2.55-3.98)$ & $3.49(2.67-4.27)$ & 0.143 \\
\hline Lymphocyte count $\left(* 10^{9} / \mathrm{L}\right)$, median (IQR) & $1.61(1.26-1.91)$ & $1.58(1.26-1.86)$ & $1.80(1.26-2.17)$ & 0.057 \\
\hline NLR, median (IQR) & $2.1(1.6-2.7)$ & $2.1(1.6-2.7)$ & $2.1(1.5-2.8)$ & 0.911 \\
\hline Platelet count $\left(* 10^{9} / \mathrm{L}\right)$, median (IQR) & $258(216-310)$ & $267(218-324)$ & $223(194-269)$ & 0.003 \\
\hline Hemoglobin (g/L), median (IQR) & $135(125-145)$ & $135(126-144)$ & $135(122-145)$ & 0.781 \\
\hline \multicolumn{5}{|l|}{ C-reactive protein } \\
\hline$<10.00 \mathrm{mg} / \mathrm{L}$ & $261(85.0)$ & $217(84.4)$ & $44(88.0)$ & 0.518 \\
\hline$\geq 10.00 \mathrm{mg} / \mathrm{L}$ & $46(15.0)$ & $40(15.6)$ & $6(12.0)$ & 0.518 \\
\hline Median length of symptoms onset to admission (days) (IQR) & $12(8-16)$ & $12(8-15)$ & $13(6-21)$ & 0.679 \\
\hline Median length of temporary hospital stay (days) (IQR) & $14(10-19)$ & $14(10-19)$ & $15(11-21.5)$ & 0.322 \\
\hline
\end{tabular}

IQR: interquartile range; NLR: neutrophil to lymphocyte ratio; CV: cardiovascular; CHD: coronary heart disease.

mild COVID-19 after adjustment for cofounders by models 1 , 2 , and 3 .

\section{Discussion}

Since the incidence of CVD is steadily increasing in China for decades $[18,19]$, many hospitalized patients might naturally have comorbid CV conditions, and recent observations show that patients with coexisting CV conditions are susceptible to the most adverse complications among patients with severe COVID-19 diseases $[6,16,20,21,22]$. However, the impact of CV conditions on the outcome of mild COVID-19 patients is limited. The present study demonstrates that comorbid CV condition is an independent risk factor for the development of severe COVID-19 and requirement for intensive care among patients with mild COVID-19. 
TABLE 2: Clinical characteristics of patients with COVID-19 with or without CV conditions.

\begin{tabular}{|c|c|c|c|c|}
\hline Characteristic & $\begin{array}{l}\text { All patients } \\
(n=332)\end{array}$ & $\begin{array}{l}\text { Without CV conditions } \\
\quad(n=284,85.5 \%)\end{array}$ & $\begin{array}{l}\text { With CV conditions } \\
\quad(n=48,14.5 \%)\end{array}$ & $P$ value \\
\hline \multicolumn{5}{|l|}{ Sex } \\
\hline Male, $n(\%)$ & $132(39.8)$ & $110(38.7)$ & $22(45.8)$ & 0.285 \\
\hline Female, $n(\%)$ & $200(60.2)$ & $174(61.3)$ & $26(54.2)$ & 0.285 \\
\hline Age, median (IQR) & $51(40-59)$ & $50(39-59)$ & $56(45-62)$ & 0.007 \\
\hline \multicolumn{5}{|l|}{ Symptoms } \\
\hline Fever, $n(\%)$ & $177(63.2)$ & $150(63.3)$ & $27(58.7)$ & 0.487 \\
\hline Cough, $n(\%)$ & $108(38.4)$ & $88(37.6)$ & $20(42.6)$ & 0.525 \\
\hline Fatigue, $n(\%)$ & $39(13.9)$ & $26(11.1)$ & $13(28.3)$ & 0.002 \\
\hline Chest tightness, $n(\%)$ & $32(11.5)$ & $14(6.0)$ & $18(40)$ & $<0.001$ \\
\hline Diarrhea, $n(\%)$ & $11(3.9)$ & $9(3.8)$ & $2(4.3)$ & 0.873 \\
\hline Headache, $n(\%)$ & $7(2.5)$ & $5(2.1)$ & $2(4.3)$ & 0.323 \\
\hline Sore throat, $n(\%)$ & $4(1.4)$ & $4(1.7)$ & $0(0.0)$ & 1.000 \\
\hline Rhinorrhea, $n(\%)$ & $4(1.4)$ & $4(1.7)$ & $0(0.0)$ & 1.000 \\
\hline Myalgia, $n(\%)$ & $12(4.3)$ & $6(2.6)$ & $6(13.0)$ & 0.001 \\
\hline Toothache, $n(\%)$ & $2(0.6)$ & $1(0.4)$ & $1(2.1)$ & 0.269 \\
\hline \multicolumn{5}{|l|}{ Comorbidities } \\
\hline Diabetes, $n(\%)$ & $11(3.3)$ & $7(2.5)$ & $4(8.3)$ & 0.036 \\
\hline Lung disease, $n(\%)$ & $7(2.1)$ & $3(1.1)$ & $4(8.3)$ & 0.010 \\
\hline Radiologic abnormality, $n$ (\%) & $201(65.5)$ & $182(67.2)$ & $19(52.8)$ & 0.088 \\
\hline \multicolumn{5}{|l|}{ Positive SARS-CoV-2 testing by RT-PCR } \\
\hline Twice or more, $n(\%)$ & $72(23.0)$ & $60(22.0)$ & $12(35.1)$ & 0.147 \\
\hline Once, $n(\%)$ & $241(77.0)$ & $216(78.0)$ & $25(64.9)$ & 0.147 \\
\hline \multicolumn{5}{|l|}{ Laboratory findings } \\
\hline Red blood cell count $\left(* 10^{12} / \mathrm{L}\right)$, median (IQR) & $4.39(4.05-4.73)$ & $4.37(4.04-4.68)$ & $4.49(4.15-4.90)$ & 0.159 \\
\hline White cell count $\left(* 10^{9} / \mathrm{L}\right)$, median (IQR) & $5.25(4.49-6.37)$ & $5.25(4.49-6.37)$ & $5.00(4.46-6.35)$ & 0.677 \\
\hline Neutrophil count $\left(* 10^{9} / \mathrm{L}\right)$, median (IQR) & $3.21(2.57-4.08)$ & $3.23(2.59-4.10)$ & $3.06(2.53-3.91)$ & 0.468 \\
\hline Lymphocyte count $\left(* 10^{9} / \mathrm{L}\right)$, median (IQR) & $1.61(1.26-1.91)$ & $1.58(1.27-1.91)$ & $1.72(1.23-1.92)$ & 0.745 \\
\hline NLR, median (IQR) & $2.06(1.60-2.67)$ & $2.07(1.60-2.67)$ & $2.04(1.56-2.54)$ & 0.609 \\
\hline Platelet count $\left(* 10^{9} / \mathrm{L}\right)$, median (IQR) & $258(216-310)$ & $263(217-314)$ & $243(202-287)$ & 0.085 \\
\hline Hemoglobin (g/L), median (IQR) & $135(125-145)$ & $135(125-145)$ & $137(130-145)$ & 0.254 \\
\hline \multicolumn{5}{|l|}{ C-reactive protein } \\
\hline$<10.00 \mathrm{mg} / \mathrm{L}$ & $261(85.0)$ & $230(85.8)$ & $31(79.5)$ & 0.300 \\
\hline$\geq 10.00 \mathrm{mg} / \mathrm{L}$ & $46(15.0)$ & $38(14.2)$ & $8(20.5)$ & 0.300 \\
\hline Median length of symptom onset to admission (days) (IQR) & $12(8-16)$ & $12(8-15)$ & $14(8-18)$ & 0.222 \\
\hline Median length of temporary hospital stay (days) (IQR) & $14(10-19)$ & $14(10-19)$ & $15(8-20)$ & 0.837 \\
\hline \multicolumn{5}{|l|}{ Clinical outcomes } \\
\hline Discharge, $n(\%)$ & $274(82.5)$ & $249(87.6)$ & $25(52.1)$ & $<0.001$ \\
\hline Require intensive care, $n(\%)$ & $58(17.5)$ & $35(12.4)$ & $23(47.9)$ & $<0.001$ \\
\hline
\end{tabular}

IQR: interquartile range; NLR: neutrophil to lymphocyte ratio.

In the present study, among hospitalized patients with mild COVID-19, fever, cough, and fatigue were the most common symptoms, which is consistent with previous reports $[14,16]$. Notably, in comparison with patients without CV conditions, patients with CV conditions showed significantly higher rates of chest tightness, fatigue, and myalgia. These finding suggests that patients with comorbidities had more severe symptoms, which clearly overlapped with the clinical symptoms of cardiovascular diseases. In the process of clinical practice, cautious screening is required to determine whether the symptoms are caused by COVID19 or progression of underlying diseases. Particularly, chest tightness and fatigue might be associated with $\mathrm{CV}$ conditions including heart failure. Therefore, SARS-CoV-2 may induce and complicate the typical symptoms and manifestations of acute myocardial infarction and chronic heart failure. 

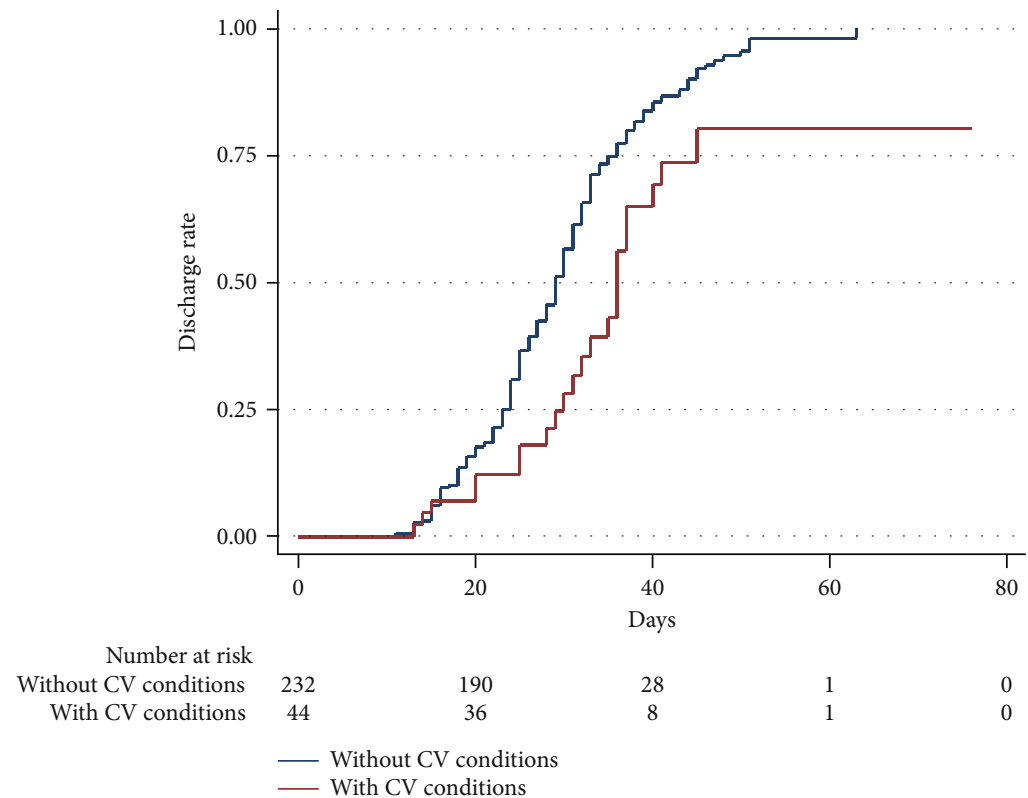

(a) Time from symptom onset

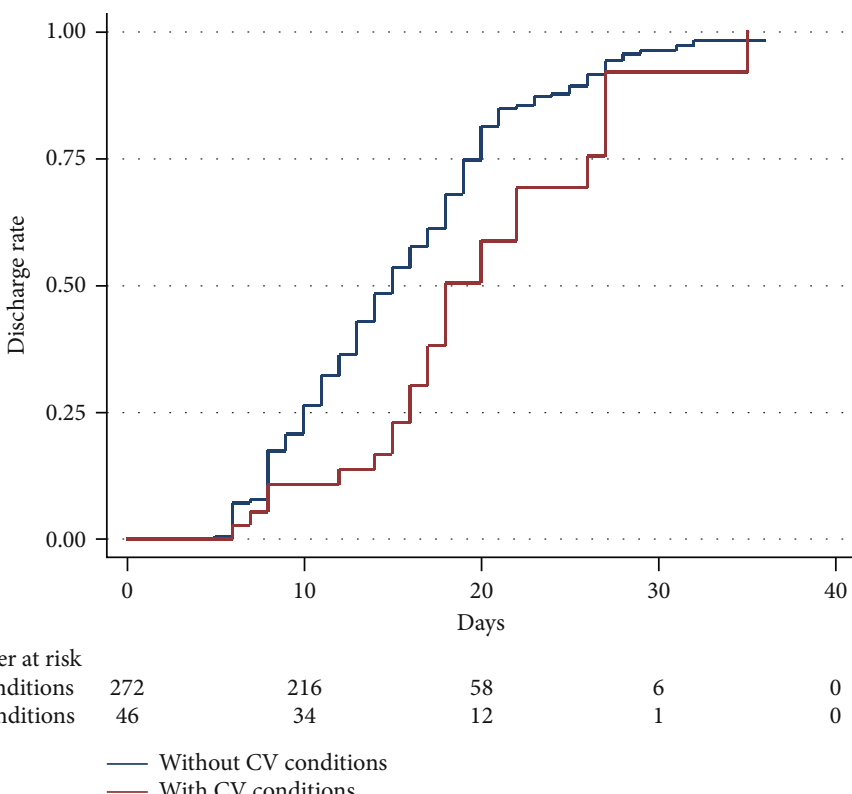

(b) Time from admission

\begin{tabular}{|c|c|c|c|c|c|c|c|}
\hline \multirow[t]{2}{*}{ Groups } & \multirow[t]{2}{*}{$\begin{array}{l}\text { No. of events/ } \\
\text { no. of patients }\end{array}$} & $\begin{array}{l}\text { Time from symptom onset } \\
\text { to referral }\end{array}$ & \multirow[t]{2}{*}{$\begin{array}{c}P \text { value } \\
\text { log-rank }\end{array}$} & \multirow[t]{2}{*}{ Groups } & \multirow[t]{2}{*}{$\begin{array}{l}\text { No. of events/ } \\
\text { no. of patients }\end{array}$} & Time from admission to referral & \multirow[t]{2}{*}{$\begin{array}{c}P \text { value } \\
\text { log-rank }\end{array}$} \\
\hline & & Duration, mean (range), d & & & & Duration, mean (range), $\mathrm{d}$ & \\
\hline Without CVD & $30 / 232$ & $31(12-58)$ & $P<0.001$ & Without CVD & $33 / 272$ & $19(11-36)$ & $P<0.001$ \\
\hline With CVD & $22 / 44$ & $30(6-76)$ & & With CVD & $23 / 46$ & $11(2-26)$ & \\
\hline
\end{tabular}

(c) Comparisons of outcomes

Figure 2: (a, b) Kaplan-Meier curves for discharge alive rate during the time from symptom onset and admission in the temporary hospital. (c) Patients with CV conditions had a lower rate of discharge alive in log-rank test, both from symptom onset and from admission. The duration means that the time from symptom onset to referral to the designated hospital for intensive care and admission in the temporary hospital to referral to the designated hospital for intensive care.

Nationwide retrospective study showed that CVD was the most common coexisting condition in patients hospitalized with COVID-19 [22]. A meta-analysis of six studies including 1527 patients with COVID-19 examined the prev- alence of $\mathrm{CV}$ conditions and reported the prevalence of hypertension, cardiac and cerebrovascular disease, and diabetes to be $17.1 \%, 16.4 \%$, and $9.7 \%$, respectively [23]. In the present study, $13.31 \%$ patients with mild cases had CV 


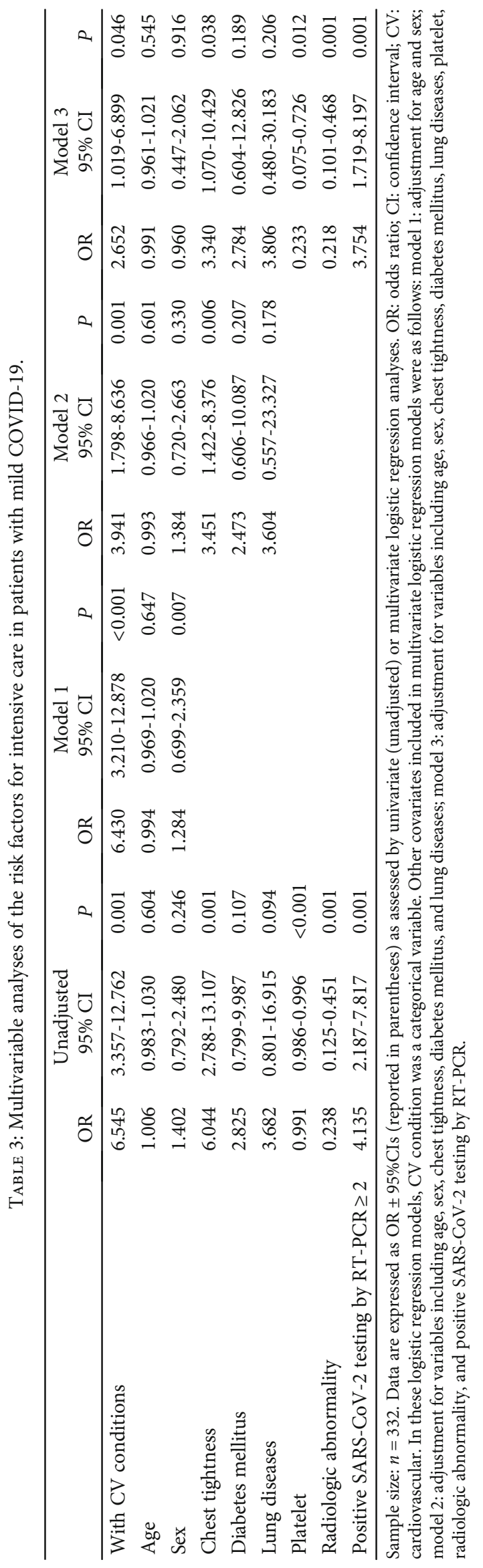


conditions. Compared to discharged patients, patients with progressive disease progression and referred to a designated hospital for intensive care were more likely to have coexisted $\mathrm{CV}$ conditions. Multivariate regression analysis suggested that $\mathrm{CV}$ condition was an independent risk factor for patients developing severe disease after adjustment of various cofounders (Table 3 ). In subgroup analysis, although mild COVID-19 patients with CHD had less positive radiologic findings than patients without CHD on admission, they still faced higher risk of unfavorable disease progression as compared to patients without CHD. Regarding the possible mechanisms, prevalent $\mathrm{CHD}$, as an inflammation disease, may serve as a marker of accelerated immunologic dysregulation and thus aggravate the immunological imbalance induced by COVID-19. On the other hand, COVID-19 itself might aggravate the myocardial injury; recent studies from Wuhan demonstrated that severe COVID-19 patients with the most ominous outcome had significantly higher TnI or TnT levels as compared to severe COVID-19 patients undergoing recovery $[24,17]$. It is known that SARS-CoV-2 can elicit the intense release of multiple cytokines and chemokines that can not only lead to vascular inflammation and plaque instability but also to myocardial inflammation [6, 19], which might be linked with the enhanced myocardial injury during the disease process. The interaction between coexisted CHD and the harm of SARS-CoV-2 on myocardial tissue might thus imply some of the mechanisms responsible for the worse outcome among mild COVID-19 patients hospitalized.

Among patients with mild cases, hypertension was the most common comorbidity, followed by CHD. An early analysis of underlying clinical features in 41 COVID-19 fatal cases revealed the highest prevalence of hypertension $(60.9 \%)$ [6]. Higher expression of ACE2 in patients with hypertension has been postulated to enhance susceptibility to SARS-CoV2 [25], although the data are conflicting and without clear suggestion implication for treatment [26]. Additional study is needed to understand the potential mechanistic relationships between hypertension and COVID-19 outcomes.

\section{Limitations}

Our study has several limitations. First, restricted by the limited medical resources, laboratory biochemical testing was insufficient; second, regarding the time of disease onset in patients, the uncertainty of the exact dates (recall bias) might have inevitably affected our assessment; lastly, data from the earliest hospitalized patients could not be fully accessed.

\section{Clinical Implication}

Mild COVID-19 patients with coexisting CV conditions should receive more intensive monitoring to prevent the transition to severe disease progression.

\section{Conclusions}

Although most mild COVID-19 patients could be discharged, approximately half of the mild COVID-19 patients with comorbid CV conditions may progress to severe disease status and require intensive care; special monitoring on these patient populations is essential to improve the outcome of these patients.

\section{Data Availability}

The data used to support the findings of this study are included within the supplementary information file.

\section{Additional Points}

Key Points. Question. What is the impact of coexisting cardiovascular $(\mathrm{CV})$ diseases on disease progression in patients with mild COVID-19? Findings. Although most patients with mild COVID-19 were discharged alive from the hospital, approximately $47.9 \%$ patients with coexisting CV conditions developed severe disease status and required intensive care. $\mathrm{CV}$ condition is an independent risk factor of intensive care among patients with mild COVID-19. Meaning. Coexisting $\mathrm{CV}$ conditions are associated with unfavorable outcomes among patients with mild COVID-19. Special monitoring is required for these patients to improve their outcome.

\section{Disclosure}

The funder had no role in the design and conduct of the study; collection, management, analysis, and interpretation of the data; preparation, review, or approval of the manuscript; and decision to submit the manuscript for publication.

\section{Conflicts of Interest}

The authors declare that they have no conflicts of interest.

\section{Authors' Contributions}

Dr. S. Zhou and Dr. J. Xu had full access to all of the data in the study and take responsibility for the integrity of the data and the accuracy of the data analysis. S. Tai, J. Tang, B. Yu, L. Tang, Y. Wang, X. Chai, and S. Zhou were responsible for concept and design. S. Tai, J. Tang, B. Yu, L. Tang, and S. Zhou were responsible for the acquisition, analysis, or interpretation of data. S. Tai, J. Sun, L. Tang, and S. Zhou were responsible for the drafting of the manuscript. Y. Wang, $\mathrm{H}$. Zhang, K. Xiao, C. Wen, C. Tan, Z. Jiang, and C. Jiang were responsible for the critical revision of the manuscript for important intellectual content. S. Tai, J. Sun, L. Zhu, and L. Jiang were responsible for statistical analysis. S. Tai and S. Zhou obtained funding. Q. Liu, Z. Fang, X. Hu, X. Li, Z. Zhu, H. Yang, T. Tao, Y. Xiao, M. Chen, and Y. He were responsible for administrative, technical, or material support. $\mathrm{X}$. Chai, J. Xu, and S. Zhou were responsible for supervision.

\section{Acknowledgments}

This research was supported by grants from the Natural Science Foundation of China (81670269 to Shenghua Zhou and 81801394 to Shi Tai). 


\section{Supplementary Materials}

Supplemental Table 1: subgroup analysis of patients with and without hypertension or CHD. Supplemental Table 2: univariable analyses of the risk factors between the stable group and the intensive group. Supplemental Figure 1: discharge rate between patients with and without hypertension (HTN). Supplemental Figure 2: discharge rate between patients with and without CHD. (Supplementary Materials)

\section{References}

[1] N. Zhu, D. Zhang, W. Wang et al., "A novel coronavirus from patients with pneumonia in China, 2019," The New England Journal of Medicine, vol. 382, no. 8, pp. 727-733, 2020.

[2] WHO, "Director-General's opening remarks at the media briefing on COVID-19 - 11 March 2020: World Health Organization".

[3] Novel Coronavirus Pneumonia Emergency Response Epidemiology Team, "The epidemiological characteristics of an outbreak of 2019 novel coronavirus diseases (COVID-19) in China," Zhonghua Liu Xing Bing Xue Za Zhi, vol. 41, no. 2, pp. 145-151, 2020.

[4] World Health Organization, "Clinical management of severe acute respiratory infection (SARI) when COVID-19 disease is suspected: interim guidance," 2020.

[5] W. J. Guan, Z. Y. Ni, Y. Hu et al., "Clinical characteristics of coronavirus disease 2019 in China," The New England Journal of Medicine, vol. 382, no. 18, pp. 1708-1720, 2020.

[6] C. Huang, Y. Wang, X. Li et al., "Clinical features of patients infected with 2019 novel coronavirus in Wuhan, China," The Lancet, vol. 395, no. 10223, pp. 497-506, 2020.

[7] H. Zhu, J. W. Rhee, P. Cheng et al., "Cardiovascular complications in patients with COVID-19: consequences of viral toxicities and host immune response," Current Cardiology Reports, vol. 22 , no. 5,2020 .

[8] L. T. Cowan, P. L. Lutsey, J. S. Pankow, K. Matsushita, J. Ishigami, and K. Lakshminarayan, "Inpatient and outpatient infection as a trigger of cardiovascular disease: the ARIC study," Journal of the American Heart Association, vol. 7, no. 22, article e009683, 2018.

[9] M. Madjid, P. Safavi-Naeini, S. D. Solomon, and O. Vardeny, "Potential effects of coronaviruses on the cardiovascular system," JAMA Cardiology, 2020.

[10] J. F. Dhainaut, Y. E. Claessens, J. Janes, and D. R. Nelson, "Underlying disorders and their impact on the host response to infection," Clinical Infectious Diseases, vol. 41, Supplementary_7, pp. S481-S489, 2005.

[11] F. Zhou, T. Yu, R. Du et al., "Clinical course and risk factors for mortality of adult inpatients with COVID-19 in Wuhan, China: a retrospective cohort study," The Lancet, vol. 395, no. 10229, pp. 1054-1062, 2020.

[12] S. M. Chen, Z. J. Zhang, J. T. Yang et al., "Fangcang shelter hospitals: a novel concept for responding to public health emergencies," The Lancet, vol. 395, no. 10232, pp. 1305$1314,2020$.

[13] Guidance for Corona Virus Disease 2019, Prevention, Control, Diagnosis and Management, People's Medical Publishing House, 2020.

[14] J. Stewart, G. Manmathan, and P. Wilkinson, "Primary prevention of cardiovascular disease: a review of contemporary guidance and literature," JRSM Cardiovascular Disease, vol. 6, 2017.

[15] WHO, "Cardiovascular diseases (CVDs)," http://www.who .int/mediacentre/factsheets/fs317/en/ (accessed 10 October 2016).

[16] T. J. Guzik, S. A. Mohiddin, A. Dimarco et al., "COVID-19 and the cardiovascular system: implications for risk assessment, diagnosis, and treatment options," Cardiovascular Research, 2020.

[17] T. Guo, Y. Fan, M. Chen et al., "Cardiovascular implications of fatal outcomes of patients with coronavirus disease 2019 (COVID-19)," JAMA Cardiology, 2020.

[18] R. O. Bonow, G. C. Fonarow, P. T. O'Gara, and C. W. Yancy, "Association of coronavirus disease 2019 (COVID-19) with myocardial injury and mortality," JAMA Cardiology, 2020.

[19] C. Yang and Z. Jin, "An acute respiratory infection runs into the most common noncommunicable epidemic-COVID-19 and cardiovascular diseases," JAMA Cardiology, 2020.

[20] N. Chen, M. Zhou, X. Dong et al., "Epidemiological and clinical characteristics of 99 cases of 2019 novel coronavirus pneumonia in Wuhan, China: a descriptive study," The Lancet, vol. 395, no. 10223, pp. 507-513, 2020.

[21] D. Wang, B. Hu, C. Hu et al., "Clinical characteristics of 138 hospitalized patients with 2019 novel coronavirus-infected pneumonia in Wuhan, China," Journal of the American Medical Association, vol. 323, no. 11, pp. 1061-1069, 2020.

[22] Z. Wu and J. M. McGoogan, "Characteristics of and important lessons from the coronavirus disease 2019 (COVID-19) outbreak in China," Journal of the American Medical Association, vol. 323, no. 13, pp. 1239-1242, 2020.

[23] B. Li, J. Yang, F. Zhao et al., "Prevalence and impact of cardiovascular metabolic diseases on COVID-19 in China," Clinical Research in Cardiology, vol. 109, no. 5, pp. 531-538, 2020.

[24] S. Shi, M. Qin, B. Shen et al., "Association of cardiac injury with mortality in hospitalized patients with COVID-19 in Wuhan, China," JAMA Cardiology, 2020.

[25] A. H. J. Danser, M. Epstein, and D. Batlle, "Renin-angiotensin system blockers and the COVID-19 pandemic: at present there is no evidence to abandon renin-angiotensin system blockers," Hypertension, vol. 75, no. 6, pp. 1382-1385, 2020.

[26] E. Driggin, M. V. Madhavan, B. Bikdeli et al., "Cardiovascular Considerations for Patients, Health Care Workers, and Health Systems During the COVID-19 Pandemic," Journal of the American College of Cardiology, vol. 75, no. 18, pp. 23522371,2020 . 